\title{
Internal Mammary to Pulmonary Artery Fistula Presenting as Early Recurrent Angina after Coronary Bypass
}

\author{
Alexandre C. Ferreira, Eduardo de Marchena, Michelle Liester, Afolabi O. Sangosanya \\ Miami, FL - USA
}

A 50-year-old man developed recurrent angina 1 year after coronary artery bypass surgery. The patient was found to have a large fistula involving branches of the internal mammary artery graft and the left pulmonary artery. In the absence of another clear cause for the patient's symptoms, we speculated that our patient's angina and abnormal stress nuclear study were due to coronary steal. In patients with a history of coronary bypass grafting, fistula formation between graft and native vessels should be considered as a possible cause of early recurrent angina.

Fistulas involving the internal mammary artery graft are rare but well-known complications of coronary artery bypass surgery. Patients with recurrent angina after surgery are usually investigated for progressive disease of the native coronary artery or the bypass graft. Fistulas involving the internal mammary artery and the pulmonary artery can be demonstrated by angiography and are rare causes of recurrent angina after coronary bypass surgery.

\section{Case report}

A 50-year-old man was admitted for evaluation of recurrent angina, occurring on exertion and at rest. Approximately 1 year earlier, he underwent coronary artery bypass grafting that included a left internal mammary artery graft to the left anterior descending artery and saphenous vein graft to the diagonal and obtuse marginal branches, and posterior descending artery. The surgery had significant postoperative complications attributed to coagulopathy.

On the physical examination, his blood pressure was 129/94. No jugular venous distention was present. The lungs were clear. No thrills were palpated over the precor-

University of Miami - Cardiovascular Center

Mailing address: Alexandre Cesar Ferreira - P.O. Box 016960 - Miami, FL - 33101 - USA Received for publication on $5 / 16 / 01$

Accepted on $7 / 11 / 01$ dium, and no murmurs or gallops were heard upon auscultation of the chest. A thallium stress test demonstrated anterior wall ischemia. Cardiac catheterization was performed and revealed native three-vessel coronary artery disease. The saphenous vein grafts to the diagonal and obtuse marginal branches and posterior descending artery were patent. The left internal mammary artery graft to the left anterior descending artery was patent. A fistulous connection emanating from the left internal mammary artery to the left pulmonary artery was visualized (fig. 1,2). A long first intercostal branch was seen proximal to the left internal mammary artery and 2 other branches that supplied flow to a vascular plexus with a fistulous connection into the pulmonary artery. The distal left anterior descending artery was only partially filled secondary to significant competitive flow to the fistula. Ventriculography revealed anterior and apical wall motion abnormalities. Blood sampling distal and proximal to the fistula showed a left-to-right shunt with a step up in oxygen saturation from the main pulmonary artery $(77 \%)$ to the distal left pulmonary artery $(81.7 \%)$. Left ventricular end diastolic pressure was elevated $(22 \mathrm{mmHg})$, and the right-sided pressure and resistance measurements were normal. Surgical correction by occlusion of the left internal mammary artery side branches supplying the fistulous connection was the initial recommendation. However, the patient preferred to try medical therapy, which to date has provided relief of symptoms.

\section{Discussion}

Several etiologies have been proposed for the formation of left internal mammary artery-to-pulmonary artery fistula. Fistulas have occurred more often in patients in whom the left internal mammary artery side branches were electrocoagulated instead of ligated ${ }^{1}$. In 1 previous case report, a large fistulous tract occurred between an unligated branch of the left internal mammary artery and the pulmonary circulation ${ }^{2}$. Disruption of the visceral pleura, along with parenchyma damage, preexisting pulmonary abnormalities, or actual pulmonary infection, have been thought to cause a 


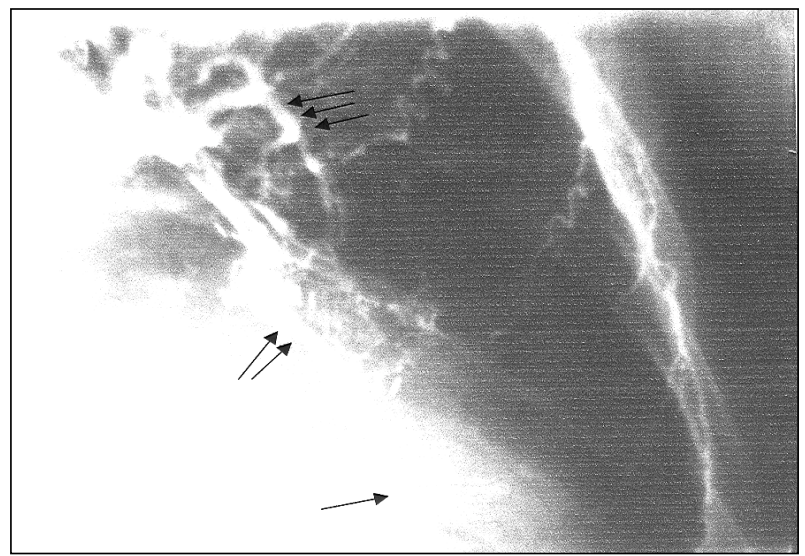

Fig. 1 - Right-oblique-cranial projection after left internal mammary injection demonstrating the native left anterior descending (single arrow), the mammary graft (double arrows), and the large fistula connection to the pulmonary artery (triple arrows).

predisposition to the development of fistulas ${ }^{3}$. In our patient, we believe that an incompletely ligated left internal mammary artery side branch was the culprit vessel in establishing the fistulous connection. Furthermore, the severe postoperative bleed that was attributed to postoperative coagulopathy could have resulted from lung or pleural trauma that lead to neovascularization and fistula formation.

The diagnosis of left internal mammary artery to pulmonary artery fistula requires cardiac catheterization. Failure to perform selective left internal mammary artery injection has resulted in failure to diagnose this complication ${ }^{4}$. The hemodynamic significance of the fistula has been assessed using Doppler-tipped guide wire and quantitative angiography ${ }^{5}$. In 1 report, left-to-right shunt was demonstrated using a first-pass radionuclide technetium- $99 \mathrm{~m} \mathrm{study}^{6}$. We were able to demonstrate a significant step up in oxygen saturation at different levels of the pulmonary artery. This method appears to be mostly effective in patients with large and more proximal fistulas.

Surgical treatment is usually recommended to prevent complications of a fistula, such as heart failure, infective endocarditis, aneurysmal expansion, and rupture and distal is-

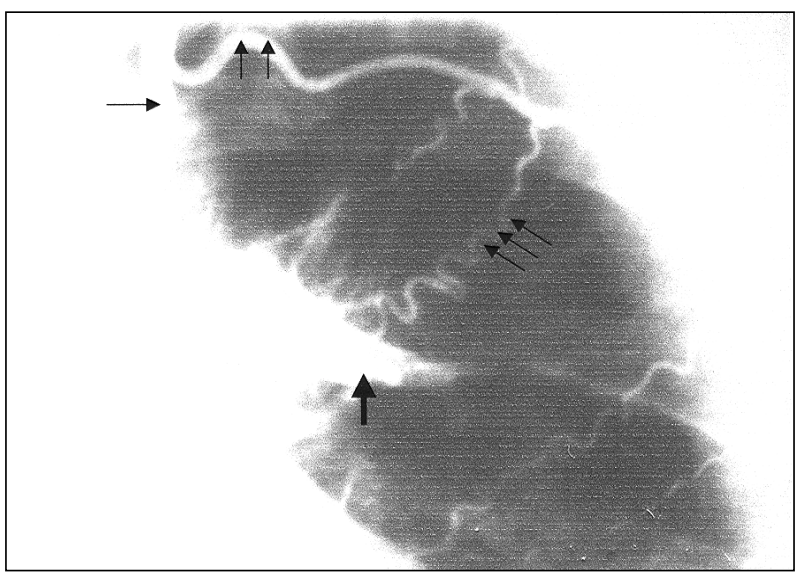

Fig. 2 - Anterior-posterior projection after left internal mammary injection demonstrating the internal mammary (single arrow), the unligated first intercostal branch (double arrows), the fistula connection to the pulmonary artery (triple arrows), and the pulmonary artery (wide single arrow).

chemia $^{7}$. Surgical management includes division of the fistula through thoracotomy. Medical therapy has been proved effective in patients who were not candidates for surgical correction ${ }^{8}$. Our patient was reluctant to have surgical correction or selective embolization. He has been successfully treated with antianginal medication and has remained asymptomatic for 16 months.

Preventing this potentially lethal condition seems to be the most advisable approach. We recommend meticulous ligation of all side branches of the left internal mammary artery pedicle before grafting. Another option is covering the left internal mammary artery graft with a pericardial flap to prevent fistula formation. However, the efficacy of this technique remains to be proven ${ }^{9}$. A fistula arising from a high branch of the left internal mammary artery such as in our patient will be less likely to be prevented by this approach.

Internal mammary artery fistulas should be considered as a possible cause of recurrent angina after bypass surgery. Coronary angiography with selective injection of the left internal mammary artery should be performed to diagnose this condition.

\section{References}

1. Wood MK. Internal mammary artery to lung parenchyma fistula(Letter). Ann Thorac Surg 1992; 54: 603.

2. Kimmelstiel Cd, Udelson J, Salen D, et al. Recurrent angina due to a left internal mammary-to-pulmonary artery fistula. Am Heat J 1993; 125: 234-6.

3. Nellens P, Stevens C, Vesrtraeten J, Heyndrickx GR. Internal mammary to pulmonary artery fistula associated with healed tuberculosis. Acta Cardiol 1980; 35: 55-61.

4. Johnson JA, Schmaltz R, Landreneau R, et al. Internal mammary artery graft to pulmonary vasculature fistula: a cause of recurrent angina. Ann Thorac Surg 1990; 50: $297-8$.

5. Reis SE, Gloth ST, Brinker JA. Assessment of the hemodynamic significance of a left internal mammary artery graft-pulmonary artery shunt in a post-bypass patient using a Doppler-tipped guide wire. Cathet Cardiovasc Diagn 1993; 29: 52-6.

6. Rashkow A, Prokop E, Fazzone P. Systemic pulmonary artery fistula diagnosed with aid of radionuclide angiography. Am Heart J 1984; 108: 1562-5.

7. Shirkey AL, Baay JE, Law SW, DeBakey ME. Arteriovenous fistula of the internal mammary vessels: report of a case and review of the literature. J Thorac Cardiovasc Surg 1964; 48: 49-53.

8. Blanche C, Eigler N, Bairey CN. Internal mammary artery to lung parenchyma fistula after aortocoronary bypass grafting. Ann Thorac Surg 1991; 52: 141-2.

9. Blanche C. Internal mammary to lung parenchyma fistula. Reply (Letter). Ann Thorac Surg 1992; 54: 603 